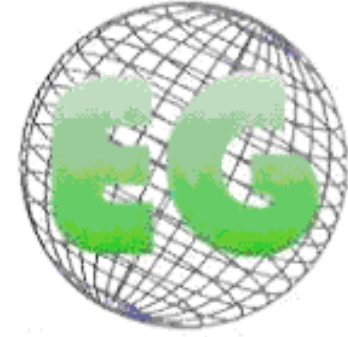

ISSN 1695-6141 N028

www.um.es/egloball

\title{
ENSAYOS
}

\section{La fenomenología para el estudio de la experiencia de la gestación de alto riesgo}

The phenomenology of the study of the experience of high-risk pregnancy

\author{
*Laza Vásquez, C., **Pulido Acuña, GP., ***Castiblanco Montañez, RA. \\ *Master in Nursing. Instructor associated-Research división. E-mail: claza1@fucsalud.edu.co \\ **Nurse. Specialist in Autonomous Learning and Computing for Teaching. Pedagogical coordinator- \\ College of nursing. ${ }^{* * *}$ Project coordinator Research división. Fundación Universitaria de Ciencias de \\ la Salud. Colombia.
}

Este artículo hace parte del proyecto de investigación No. 55-17692 " Experiencias vividas por mujeres gestantes con preeclampsia severa atendidas en el Hospital San José-Bogotá: un estudio cualitativo" financiado por Fundación Universitaria de Ciencias de la Salud-Hospital de San José.

Palabras clave: Fenomenología descriptiva; investigación cualitativa; vivencias; experiencias; gestación de alto riesgo.

Keywords Descriptive phenomenology; qualitative research; experiences; high-risk pregnancy.

\section{RESUMEN}

La investigación cualitativa capta la realidad social a través de los ojos de los seres humanos, mediante un enfoque que permite la amplitud, flexibilidad y profundidad. Dentro de ella, la Fenomenología descriptiva da cuenta de las experiencias humanas sobre fenómenos vividos, descritos en primera persona y presentados en estructuras de significados. Esto se ha convertido en una importante contribución al pensamiento y desarrollo enfermero; por lo anterior, en este artículo se reflexiona acerca de la utilidad de la tradición para el abordaje de las vivencias de las mujeres durante una gestación de alto riesgo. Para esto, se presentan unas generalidades de la Fenomenología y de resultados de investigación científica del fenómeno propuesto en este artículo de reflexión.

Se resalta la conveniencia del abordaje fenomenológico ya que aproximarse a la vivencia durante una gestación de alto riesgo permite evaluar las necesidades objetivas y subjetivas del cuidado de las mujeres y sus familias durante la atención prenatal, y la hospitalización durante el parto y el puerperio; permite profundizar en las relaciones establecidas entre las gestantes y los profesionales, las alteraciones que causa en la familia y las potencialidades que esta puede desarrollar; como acercarse a las emociones y sentimientos de las gestantes. Así, se concluye que es imperioso acercarse desde este referente, ya que mediante la interacción con las gestantes y sus experiencias de vida se propicia 
una reflexión que aportará a mejorar la calidad en el proceso de cuidado de enfermería, más allá de una dimensión biológica.

\begin{abstract}
Qualitative research captures social reality through humane eyes, though an ample, flexible and profound approach. Inside, descriptive phenomenology accounts on human experiences of perceived phenomena, told in first person and presented as structures of meaning. This has turned into an important contribution to the ways of thought and development of nursery; therefore, there is an insight in this paper about the tradition to deal with past experiences of women during a high-risk pregnancy. To do this, general principles of Phenomenology and scientific research results about this phenomenon are presented.
\end{abstract}

Convenience of this phenomenological approach is highlighted in order to get closer to the past experiences during a high-risk pregnancy allows evaluation of the objective and subjective needs of women and their families during prenatal attention, and hospitalization during delivery and puerperium; it allows deep analysis of established relationships among expecting women and health professionals, changes caused to family and potentiality to be developed, and to get closer to pregnant women's emotions and feelings. So, an approach from this reference it is imperative, for an insight from interaction with expecting women and life experiences can be obtained, in order to improve nursery care quality beyond biological dimension.

\title{
INTRODUCCIÓN
}

La complejidad del ser humano y de las realidades sociales que construye y en las que se encuentra inmerso, aboca a la imperiosa necesidad del uso de un enfoque investigativo diferente al positivismo para acercarse al conocimiento científico. Así, con la diversidad de acervos originados en las Ciencias sociales, la investigación cualitativa se ha convertido en esa alternativa válida para estudiar y comprender los fenómenos humanos y sociales, ante la imposibilidad de "fraccionar, reducir o mutilar" estas realidades.

Según Bonilla y Rodríguez ${ }^{1}$, el enfoque cualitativo tiene como principal característica el interés por captar la realidad social "a través de los ojos" de la gente que está siendo estudiada, es decir, a partir de la percepción que tiene el sujeto de su propio contexto. No se parte de supuestos derivados teóricamente, sino que busca conceptualizar la realidad con base en el comportamiento de las personas estudiadas, explorando sistemáticamente los conocimientos y valores que comparten los individuos en un determinado contexto espacial y temporal. A esto se agrega que "la investigación cualitativa requiere como actitudes fundamentales la apertura, la flexibilidad, la capacidad de observación y la interacción con el grupo de investigadores y con los actores sociales envueltos". 2

Por lo anterior, además de permitir la profundización en diferentes aspectos de la realidad, este tipo de enfoque también se convierte en un recurso ideal para ampliar y complementar los resultados de la indagación cuantitativa, ya sea mediante el proceso de triangulación o de la utilización de un diseño investigativo mixto. ${ }^{3}$ En el caso de Enfermería permite obtener una visión holística, comprensiva y contextualizada de los fenómenos relacionados con los cuidados de la salud. ${ }^{4}$

Este enfoque investigativo utiliza un conjunto de "tradiciones" investigativas desde donde asume la pesquisa de sus objetos y sujetos de interés. También llamadas "estrategias de investigación", son enfoques de la investigación cualitativa que tienen una historia distinguida en una de las disciplinas de las ciencias sociales. ${ }^{5}$ Dentro de 
este abanico se encuentra la Fenomenología, considerada una orientación filosófica y metodológica que procede de la Filosofía. Existen dos escuelas de la Fenomenología: la trascendental o descriptiva y la interpretativa 0 hermenéutica. ${ }^{6}$ La primera, de interés en este artículo, tuvo como fundador a Edmund Husserl durante la primera década del siglo XX y dentro de sus representantes se citan a Maurice Merleau Ponty, Gabriel Marcel y Jean Paul Sartre. ${ }^{7}$

Este artículo pretende presentar la utilidad de la Fenomenología descriptiva para el abordaje de un fenómeno de particular interés para la Enfermería: las vivencias/experiencias de las mujeres que han vivido una gestación de alto riesgo y las diferentes implicaciones del mismo.

\section{Más allá del positivismo para la pesquisa enfermera: la Fenomenología.}

Husserl ${ }^{8}$ consideró la Fenomenología trascendental o descriptiva como "la ciencia de la esencia de la conciencia" y centró la definición en el concepto de intencionalidad y el significado de las experiencias vividas desde el punto de vista de la primera persona. Afirmó que el significado de las experiencias vividas puede ser descifrado sólo a través de las transacciones uno a uno entre el investigador y los objetos de investigación. Estas transacciones deben involucrar una atenta escucha, interacción y observación para crear representación de la realidad más sofisticada que la definida en previos entendimientos.

En otras palabras, su intención es comprender los fenómenos en sus propios términos para proporcionar una descripción de la experiencia humana tal como es experimentada por la misma persona ${ }^{9}$, o la reconstrucción del mundo interior de la experiencia del sujeto, ya que cada individuo tiene su propia manera de experimentar la temporalidad, la espacialidad, la materialidad. Pero cada una de estas coordenadas debe entenderse en relación con los demás y al interior de su mundo. ${ }^{10}$

El foco de la investigación fenomenológica son las experiencias de la persona en relación con un fenómeno. En este sentido, los expertos en esta tradición investigativa asumen que las experiencias dan sentido a la percepción de cada persona sobre un fenómeno particular; siendo su objetivo la descripción total de la experiencia y de las percepciones que ella expresa ${ }^{6}$. Lo anterior propone la representación de la estructura total de la experiencia vivida, incluyendo el significado que estas experiencias tienen para los individuos que en ellas participan, preocupándose de la compresión y no de la explicación. ${ }^{11}$

Esta tradición investigativa se ha utilizado para la incorporación de fenómenos propios de la Enfermería, dado el peso de la intersubjetividad que se establece en la relación enfermera(o)-sujetos de cuidado. Así, como afirma Tierra et al 6 , al intentar la investigación enfermera entender el significado de la experiencia de los seres humanos, en la Fenomenología se ha encontrado una importante contribución al pensamiento enfermero para la comprensión de la realidad del día a día en que se encuentra inmerso el quehacer disciplinar. Se necesita sumergirse en la subjetividad y la esencia del ser humano al que se cuida, sin olvidar la objetividad impregnada en él. En este sentido, la esencia significa la posibilidad por un lado, de una lectura de la realidad del fenómeno y la experiencia vivida, y por el otro, la subjetividad como una característica del sujeto que refuerza su aspecto humano de ser único y singular. 
Ahora bien, ¿por qué estudiar el fenómeno particular de las experiencias/vivencias de las mujeres que han vivido una gestación de alto riesgo desde éste referente filosófico y metodológico? Desde la postura de la Enfermería como disciplina y profesión, es necesario acercarse a esta problemática para comprender cómo las gestantes viven, experimentan y atribuyen significados a este fenómeno particular; así como buscar el sentido que éste tiene para ellas y los sentimientos que les generan. Es necesario aprender, en la interacción con las mujeres, sus experiencias de vida para encontrar lo que esconden. Muchos son los conflictos que puede presentar una mujer con una gestación de alto riesgo: la incertidumbre por el desenlace, la angustia y el miedo generado por su vida y la de su hijo. Así, en este proceso la conexión y relación de las mujeres con su hijo por nacer son amenazadas. Por esto, en la lucha de las mujeres por mitigar el estrés que generan las complicaciones perinatales y posterior hospitalización, se establece una búsqueda existencial de significado y del valor que puede ser intenso.

Precisamente, estas vivencias/experiencias pueden ser elaboradas, desde la investigación científica, como unidades de significados que den razón de las construcciones que hacen las gestantes acerca de este fenómeno. En términos de Jorge, Fiúza y Queiroz ${ }^{12}$, la Fenomenología permite una visión humana sobre estas vivencias. En este caso, las experiencias de las gestantes de alto riesgo, facilita el reconocimiento en cada una de ellas, siendo un ser que vive una experiencia única cuyo significado, al ser entendido, hace posible la comprensión del sentido de este evento.

Así, en un mundo medicalizado, además de la necesidad de rescatar la otra mirada, la del sujeto que vivencia y experimenta las dolencias y padecimientos; es apremiante tomar en cuenta al sujeto que padece más allá de lo biológico y lo fisiológico. Es, justamente, explorar cómo vive la enfermedad y cómo esto también influye en este proceso. En este sentido, y para la Enfermería, los estudios fenomenológicos han demostrado ser una puerta para la reflexión y la consecución de la calidad en el proceso de cuidado. ${ }^{13}$ Al buscar la conciencia y los significados a través de esta tradición, las necesidades y potencialidades de los individuos se dan a conocer mejor y se abren vías importantes para la realización de cambios en los procesos de cuidado enfermeros; y que permitan construir un conocimiento comprensivo sobre estos fenómenos particulares. ${ }^{14}$

La Fenomenología es el punto de partida, es "volver a las cosas mismas", reconociendo la prioridad de la práctica, el ámbito de las decisiones y actuar sobre el pensamiento y la reflexión. La experiencia no es sólo la capacidad humana para representar el mundo a través de los procesos cognitivos, ya que se tiene en sí misma "significaciones" o "unidades ideales significativas". Para ella, la operación primordial de significación es que no existe separadamente de la expresión y, como tal, es un fenómeno sensible al cuerpo (y no una mera cuestión de la "subjetividad"). ${ }^{13}$ De esta forma, el método fenomenológico está dirigido a fijarse en los significados que se dan, y que son tal como son propuestas por las experiencias propias.

\section{¿Cuáles son las vivencias/experiencias de las mujeres que han enfrentado una gestación de alto riesgo?}

Algunas experiencias investigativas han comprobado la validez de la Fenomenología para el acercamiento de las vivencias de las mujeres durante una gestación de alto riesgo. A continuación se presentan algunas de ellas. 
En Brasil, Souza et al ${ }^{15}$, analizaron la experiencia vivida por mujeres frente a una gestación de alto riesgo por preeclampsia. Las mujeres dieron cuenta de la falta de conocimiento e ignorancia en su alteración de salud así como de los factores relacionados con el nacimiento prematuro, en especial, la asociación de esta última con la hipertensión gestacional. En la percepción y conocimiento de la enfermedad, las mujeres citaron factores como la religión, problemas familiares y errores de la propia mujer. Este desconocimiento contribuyó a la poca búsqueda de atención preventiva y favoreció la hospitalización temprana debido a la gravedad de la mujer. Por otro lado, la experiencia de tener a su hijo hospitalizado generó sentimientos de tristeza y desesperación por el estado de gravedad y por los equipos a los que se encontraban conectados sus hijos en la Unidad de Cuidado Intensivo Neonatal (UCIN); las dificultades en la cotidianidad durante la estancia con su hijo en esta: la incapacidad de manejar a su hijo por su estado de gravedad, el miedo alimentarlo por una sonda nasogástrica, la impresión que causó la fototerapia, y los sufrimientos que generaron los diferentes procedimientos; a pesar de ser conscientes de su necesidad para la sobrevivencia de su hijo. Sin embargo, también expresaron los sentimientos de alegría y felicidad cuando lograron interactuar por primera vez con el menor: poder cargarlos, escucharlos llorar y la suspensión de los diferentes procedimientos.

Azevedo, Araújo, Costa y Medeiros ${ }^{16}$ establecieron la vivencia también en mujeres que padecieron una gestación con preeclampsia, emergiendo tres categorías: concepciones, aparición y sentimientos en relación a la dolencia. La primera evidenció percepciones del riesgo de la enfermedad relacionadas con tres aspectos: la muerte, las consecuencias de la enfermedad y la incertidumbre sobre lo que podría suceder. Las dos primeras eran percibidas dado el estrecho vínculo madre-hijo durante la vida intrauterina. La aparición de la preeclampsia tuvo una forma "inesperada" e "impredecible"; y su causalidad establecida con factores hereditarios, emocionales (ira, ansiedad y estrés); problemas con la pareja como algún tipo de violencia contra la mujer, la falta de un cuidado adecuado durante el embarazo y la aparición de hipertensión arterial. En el caso de haber sido padecida por una pariente o la misma mujer, fue definida como un evento natural. La alteración de salud generó preocupación y ansiedad por considerarla grave y algo desconocida. Sin embargo, frente a la percepción de estos riesgos, las mujeres expresaron que no fueron escuchadas por los profesionales de la salud.

También en Brasil, Souza, Cecatti, Parpinelli, Krupa y Osis ${ }^{17}$ estudiaron las experiencias de las mujeres que habían sobrevivido a complicaciones graves relacionadas con morbilidad materna severa. Los desgarradores relatos dieron cuentan de un acontecimiento inesperado y desconocido que generó sentimientos de angustia, preocupación y miedo por la inminencia de la muerte y de transitoriedad de la vida; lo cual se vio reforzada con frecuencia por la aparición de experiencias desagradables como el dolor, la disnea y frustración por la interferencia con la progresión natural de embarazo. A esto se sumó la vivencia de los cambios físicos como el aumento de peso por la retención de líquidos y el difícil proceso de tratamiento y recuperación, así como la incomodidad física asociada a la enfermedad. La enajenación apareció al evidenciar como las mujeres se sintieron distanciadas a eventos como el nacimiento de su hijo frente a procesos como su enfermedad y tratamiento. Para ellas, ese momento generó la necesidad de apoyo por parte de las personas más cercanas como su familia y su esposo o compañero sentimental.

Más desgarrador aún fueron las narraciones de las mujeres de quienes sus hijos murieron y que por su estado de salud no lograron vivir este momento. De ellas 
emergió otra categoría: "lagunas de la memoria y necesidad de información" que hizo referencia al estado de alteración de la conciencia causada por estar bajo sedación o en coma; al "despertar" se enteraron de acontecimientos significativos como la muerte o funeral de sus hijos. Estos hechos, de capital importancia, se configuraron como negativos y en muchas quedó la sensación de que una parte de su vida había desaparecido de la "película" de su existencia. Esto fue algo muy difícil de aceptar dentro de la experiencia vivida. ${ }^{17}$

Carvalheira, Tonete y Parada ${ }^{11}$, al estudiar el fenómeno anterior en una población similar, además de obtener categorías muy parecidas, encontraron que esta situación desencadenó la aparición de emociones y sentimientos de culpa ya que la morbosidad materna grave es representada como algo asociado a fallas de la propia madre durante la gestación, resultado del rechazo de su organismo al feto generando culpa. También representó un conflicto con lo inesperado, como un gran desafío a las mujeres en las que sus hijos requerían de muchos cuidados, provocando así, sufrimiento. Pero igualmente, significó una nueva experiencia en la vida de las mujeres y posibilitó la vivencia de la maternidad. Por lo anterior, el período puerperal fue identificado y descrito como difícil debido a la inestabilidad emocional y por la situación de gravedad del recién nacido. Esto también representó la necesidad de reorganizar sus vidas y los recursos económicos, lo que significó inseguridad y temor en las mujeres; además que las dificultades socioeconómicas y limitaciones en la vida cotidiana implicaron poco tiempo para el cuidado de sí mismas.

Desde otro ángulo, Price, Lake, Breen, Carson, Quinn y O' Connor ${ }^{18}$ exploraron las vivencias, desde las experiencias espirituales, como soporte durante un embarazo de alto riesgo en puérperas canadienses. La gestación significó sentimientos de miedo, incertidumbre, soledad, frustración y tristeza al plantear la posibilidad de la muerte 0 problemas de salud del hijo deseado; y la separación de las mujeres de sus familias debido a la hospitalización temprana o el reposo en cama. Por lo anterior, la experiencia gozosa de la espera se convirtió en una situación desalentadora en sus vidas, sintiéndose ansiosas y solas. Así "la búsqueda de un lenguaje espiritual", como conceptos culturales y religiosos dominantes, ayudó a comprender y articular la experiencia personal y espiritual frente a la enfermedad; aunque en muchas ocasiones no fue suficiente para responder frente a la incertidumbre de esta.

Las prácticas espirituales variaron desde tener una relación trascendente y sagrada con un ser superior para las que profesaban la fe cristiana; asistir a la iglesia, participar de la oración personal y formal, y vivir de acuerdo con los valores y creencias establecidas por su fe religiosa. Otras las definieron en términos religiosos pero sin estar conectadas a ninguna religión en particular: una relación con las almas, espíritus o energías que estaban presentes dentro y alrededor de las personas. Las ateas manifestaron su espiritualidad en términos de relaciones, emociones y recuerdos, de prácticas como el contacto con la naturaleza, expresar sus emociones, vivir momentos íntimos con sus familiares y amigos; y según sus valores morales y el diálogo espiritual. Estas acciones se asumieron como un acto de oración o diálogo consigo misma para un resultado positivo frente al padecimiento que calmó los miedos y alivió los sentimientos de soledad; lo cual ayudó a las mujeres a sentirse tranquilas, relajadas, seguras y confiadas. ${ }^{18}$

Por otro lado, varios trabajos extienden el acercamiento de estas experiencias al núcleo familiar, mostrando cómo la vivencia de las mujeres que han padecido una gestación de alto riesgo no se limita a ellas. Así, este evento provoca muchos 
cambios en las condiciones del binomio madre-hijo y también modifica las expectativas de la mujer y las familias acerca de la gestación. ${ }^{19}$

En este orden, Sittner, DeFrain y Brage $^{20}$, encontraron que la familia emergió como una célula social de apoyo y soporte, identificándose las siguientes fortalezas para enfrentar el desafío que suponía una gestación de alto riesgo: capacidad para hacer frente a la enfermedad, centrado en el apoyo social, resistencia individual y para administrar el estrés y la crisis eficazmente; mantenimiento de canales abiertos de comunicación (a través de visitas hospitalarias y llamadas telefónicas), participación de la familia en consultas prenatales, pasar el tiempo juntos y de forma agradable, manifestaciones de agradecimiento por parte de la gestante y de su familia a todos los que las apoyaron; bienestar espiritual manifestado en la fe en Dios y la iglesia como un soporte social y de orientación durante el proceso; y compromiso de la familia durante el mismo.

Por otro lado, Silva, Cordova, Chachamovich y Záchia ${ }^{21}$ identificaron las repercusiones de la preeclampsia en las gestantes y sus familias en un grupo de mujeres brasileñas. En las primeras, el padecimiento significó la pérdida de la independencia y del control en sus vidas; así como desistir en la planificación de embarazos posteriores. También implicó actividades objetivas como su autocuidado, el cuidado del hogar y de su familia, y subjetivas, como la necesidad de asumir todos los acontecimientos de forma menos traumática, realizar procesos de aceptación y rituales de luto. En las familias significó la necesidad del acompañamiento para una mayor seguridad durante la experiencia de la gestación; siendo fundamental la participación de la familia y del esposo en el proceso facilitador de la reorganización objetiva y subjetiva en la vivencia de la mujer y un mediador en situaciones de estrés.

Otro elemento presente en los relatos en las gestantes fue el impacto que causó la forzada hospitalización para la atención y cuidado dado por la amenaza sobre su vida y la del hijo por nacer. Sobre esto, Leichtentritt, Blumenthal, Elyassi, Sigi y Rotmensch $^{22}$ en un estudio realizado en mujeres hebreas con gestaciones de alto riesgo en un hospital israelí, describieron esta experiencia desde la frustración, la pérdida, la ira y la soledad que les generó. Esta se relacionó con varios eventos como la falta de un problema específico que pudiera ser tratado para ser dada de alta, la sensación de soledad y la experiencia de un fuerte aislamiento social y familiar. Además se agregó el miedo y la ansiedad asociados a la salud del feto y el temor de dar a luz a un niño con discapacidad; y la situación del resto de la familia por el abandono temporal del hogar. Junto con los sentimientos anteriores, también emergieron otros: la esperanza y la confianza en el personal sanitario y en Dios para salvar su hijo, y que este naciera sano. Finalmente, expresaron una ambivalencia en las emociones que estuvo presente durante toda su narrativa: por un lado, deseaban prolongar lo más posible su gestación por el bien de su hijo, pero por otro, anhelaban adelantar el nacimiento para terminar con esta dura etapa de su vida.

Richter, Parkes y Chaw-Kant ${ }^{23}$ en Canadá encontraron dos grandes temas expresados por las gestantes durante la hospitalización: los estresores asociados al reposo restrictivo en cama y las necesidades asociadas a este. El primero se relacionó con una "pérdida de control" sobre su embarazo y en el desempeño de las funciones y actividades cotidianas, tanto profesionales como del hogar. Además, prevalecieron sentimientos de ser una "carga" para sus familias por los cambios que suponía en sus vidas, en especial en los tema financieros, el cuidado de los hijos por parte de sus esposos y los desplazamientos de estos para visitarlas; así como 
incapacidad de autocuidarse y satisfacer sus necesidades. Dentro de las "necesidades" asociadas al reposo, expresaron las de mayor privacidad, fuente de malestar por las continuas visitas médicas y la posibilidad de que sus familiares y esposos pudieran escuchar su condición clínica. Otras, como las ocasionadas por los cambios bruscos en la dieta, de mayor información sobre su estado de salud por parte de los profesionales y de esparcimiento frente al aburrimiento de la hospitalización. Otras necesidades fueron las de un mayor contacto con sus familias y esposos; de sensibilizar más a estos últimos sobre su situación y de una atención más personalizada de la gestante y sus esposos por parte del personal.

Barlow, Hainsworth y Thornton ${ }^{24}$ también abordaron las experiencias de las mujeres, pero en este caso cuando fueron hospitalizadas de forma súbita para ser atendidas por la amenaza de un parto prematuro en un hospital del Reino Unido. Los resultados demostraron las vivencias de incertidumbre y la búsqueda de sentido al evento de la hospitalización para la atención, ya que percibieron el suceso como repentino e inesperado; y con una gran carga de incertidumbre por lo que sucedería a la vida de sus hijos dado el adelanto del nacimiento. La angustia por los síntomas físicos que experimentaron y el desconocimiento de las causas de su estado también fueron experiencias descritas por las gestantes. A esto, se le sumó el miedo al parto, tanto por la vida de su hijo como por el temor al dolor. Al final, las mujeres expresaron sentir no haber sido tomadas en cuenta por el personal sanitario que las atendió, lo que dificultó la vivencia de este evento.

Finalmente, las relaciones con el equipo de salud es un tema también estudiado y emergente en la investigación fenomenológica en las mujeres con gestaciones de alto riesgo.

Oliveira y Madeira ${ }^{25}$ analizaron la interacción entre el equipo interdisciplinario de salud y las mujeres. La información recibida por parte de los profesionales acerca de su estado fue considerada como escasa, contradictoria, poco clara; y en la cual se hacía relación al riesgo pero sin que se les explicara en qué consistía este. Además, fueron establecidas como "reglas" transmitidas de forma vertical, las cuales debían seguir para disminuir el riesgo,sin disminuir sus miedos y dudas.

Silva, Cordova, Chachamovich y Záchia ${ }^{21}$ encontraron desconocimiento en el significado de la enfermedad hipertensiva de la gestación en la mayoría de las participantes en un estudio realizado en Brasil. Las que informaron tener alguno, refirieron que la fuente de conocimiento no fueron los profesionales que las atendieron durante el control prenatal u hospitalización, sino otras como la internet. Además, cuando fueron informadas del diagnóstico por los médicos, esta fue percibida como negativa por la falta de diálogo y de orientación sobre la patología. Por lo anterior, las mujeres expresaron el deseo de una mayor atención y acompañamiento de los profesionales que les brindaron atención prenatal.

Otra mirada es la experiencia vivida por la mujer durante la hospitalización del hijo prematuro en una UCIN presentan Souza et al ${ }^{15}$. Este evento se constituyó en una prolongación de la estancia hospitalaria, y se vio agravado dado que la institución hospitalaria no contemplaba programas que favorecieran el desarrollo de actividades saludables para reducir el estrés y promover la socialización materna. Así, los recursos para satisfacer las necesidades de escucha y apoyo de la puérpera fueron los familiares, la interacción entre las madres, los provenientes de la fe y de algunos profesionales. También reveló las actitudes y relaciones de conflictos y dudas entre 
los profesionales y la mujer con su hijo en la UCIN. Aunque no existió ningún obstáculo para la participación materna en el cuidado de su hijo, estas expresaron que carecían de la capacidad para interactuar efectivamente, revelando que el personal de la UCIN no era consciente de estas dificultades. De este modo, los profesionales no proporcionaron la suficiente información y el uso de un lenguaje técnico que promovió la construcción de una realidad distorsionada.

\section{A manera de cierre}

Los resultados investigativos presentados en esta breve revisión resaltan la utilidad del enfoque cualitativo y de la Fenomenología descriptiva para el acercamiento a un tema de gran trascendencia para la Enfermería como el tratado en este escrito. De esta manera, para los profesionales, aproximarse a la experiencia vivida permitirá evaluar las necesidades de cuidado de las mujeres con gestaciones de alto riesgo y de sus familias desde la atención prenatal, y la hospitalización durante el parto y el puerperio. Lo anterior, a partir de una visión holística de la mujer, en la cual se tenga en cuenta su contexto socioeconómico y cultural.

De esta forma, la investigación fenomenológica ha dado cuenta de la necesidad de indagar otras áreas diferentes a la clínica, como los sentimientos que generan la alteración y las emociones mixtas y ambivalentes que experimentan las mujeres y sus familias; las necesidades objetivas pero también las subjetivas de las gestantes, la percepción y experiencia de la relación con los profesionales durante la atención y el cuidado recibido; las duras experiencias durante la hospitalización así como el impacto en la familia y el rol que esta juega; entre otras. El conocimiento de lo anterior mejoraría la calidad del cuidado de enfermería y ayudaría a mitigar los sentimientos de miedo, desesperanza e incertidumbre así como la aceptación de la gestación para conseguir cambios en actitudes y hábitos poco saludables. ${ }^{25}$

En tal sentido, el conocimiento obtenido podrá dar cuenta tanto del cuidado como herramienta para el "quehacer" de Enfermería como de la preocupación por "el ser" de la vivencia de las mujeres; aportando nuevos elementos al campo disciplinar. Conocer cómo se vive esta particular experiencia se podría considerar un avance, pero más aún se enriquecerá con la identificación de atributos de las categorías, los cuales varían de acuerdo con las características del fenómeno, su escenario sociocultural y socioeconómico. Estos hallazgos se requieren para evidenciar y ser utilizados en intervenciones de cuidado coherentes con la realidad vivida de las gestantes. Esta es, al mismo tiempo, una forma de aportar y fortalecer el cuerpo disciplinar, en especial, para mejorar la calidad del cuidado a las mujeres en esta situación.

Desde lo social, entendido como la interacción de las mujeres con su familia y colectivo, este enfoque permitirá aproximar la práctica de Enfermería a la realidad que viven y deben afrontar las gestantes; y a los vínculos que desarrollan o desean desarrollar. Acercamiento que puede retroalimentar a los equipos de salud para prevenir e intervenir este tipo de situaciones de salud, y de esta manera, mejorar la atención.

Todo lo anterior posibilitará la realización de un ejercicio crítico el cual aportará significativamente al pensamiento enfermero; teniendo en cuenta que para comprender la realidad cotidiana de este ejercicio, la enfermera debe sumergirse en la subjetividad y la esencia del ser humano sujeto de cuidado; llevándolo a una 
comprensión de la realidad que vivencia la persona. Además, esta tradición investigativa igualmente se convierte en una estrategia de reflexión, la cual al explorar la vivencia de la mujer durante una gestación de alto riesgo le permite interactuar de manera directa con ella, y así, diseñar procesos de cuidado que se acercan de manera directa a suplir las necesidades de la misma, permitiendo la práctica de un cuidado con calidad.

\section{REFERENCIAS}

${ }^{1}$ Bonilla $\mathrm{E}$, Rodríguez P. Más allá del dilema de los métodos: la investigación en ciencias sociales. Bogotá: editorial Norma, 2005.

${ }^{2}$ Minayo, C. El desafío del conocimiento. Investigación cualitativa en Salud. Buenos Aires: Lugar EditoriaL, 1997.

${ }^{3}$ Hanson W, CreswelL J, Plano V, Petska K, Creswell D. Mixed methods research designs in counseling psychology. Journal of Counseling. Psychology. 2005;52(2):224-235.

${ }^{4}$ Siles J, Solano C, Cibanal L. Holismo e investigación cualitativa en el marco de la antropología de la complejidad. Una reflexión sobre la pertinencia metodológica en ciencias socio-sanitarias y humanas. Cultura de los Cuidados. 2005;19(1):68-81.

${ }^{5}$ Denzin NK; Lincoln YS. Handbook of qualitive research. thousand oaks, ca: sage publications, 1994

${ }^{6}$ Terra GM, Silva LC, Camponogara S, Santos EKA, Souza AIJ, Erdmann AL. Na trilha da fenomenologia: um caminho para a pesquisa em enfermagem. Texto Contexto Enferm. 2006;15(4):672-678.

7 Palacios L. Fundamentos y desarrollo de un protocolo de investigación fenomenológica en enfermería. Enferm Intensiva. 2010;21(2):68-73.

${ }^{8}$ Husserl E. Logical investigations. New York: Humanities Press, 1970.

${ }^{9}$ Bentz VM, Shapiro JJ. Mindful enquiry in social research. Thousand Oaks, CA: Sage Publications, 1998.

10 Groenewald T. A Phenomenological research design illustrated international. Journal of Qualitative Methods. 2004;3(1):42-55.

${ }^{11}$ Carvalheira AP, Tonete VL, Parada MG. Sentimientos y percepciones de mujeres en el ciclo embarazo-puerperio que sobrevivieron a una morbosidad materna grave. Rev. Latino-Am. Enfermagem. 2010;18(6):1187-1194.

12 Jorge MSB, Fiúza GV, Queiroz MVO. La fenomenología existencial como posibilidad de comprensión de las vivencias del embarazo en adolescentes. Rev. Latino-Am. Enfermagem. 2006;14(6):907-914.

${ }^{13}$ Baptista $P$, Merighi M, Freitas G. El estudio de la fenomenología como una vía de acceso a la mejora de los cuidados de enfermería. Cultura de los cuidados. $2011 ; 29(15): 9-15$.

${ }^{14}$ Uribe C, Rivera MS, Contreras A, Bravo P, Cornejo M. Significado del bienestar materno en la experiencia de parto. Horizon enferm. 2007;18(1):89-100.

${ }^{15}$ Souza N, Araújo AC, Azevedo G, Jerônimo SM, Barbosa L, Sousa NM. Maternal perception of premature birth and the experience of pre-eclampsia pregnancy. Rev. Saúde Pública. 2007;41(5):704-710.

${ }^{16}$ Azevedo D, Araújo AC, Costa Í, Medeiros JA. Percepções e sentimentos de gestantes e puérperas sobre a pré-eclâmpsia. Rev. salud pública. 2009;11(3):347358.

${ }^{17}$ Souza JP, Cecatti JG, Parpinelli MA, Krupa F, Osis M. An emerging "maternal nearmiss syndrome": narratives of women who almost died during pregnancy and childbirth. Birth. 2009;36(2):149-158. 
${ }^{18}$ Price S, Lake M, Breen G, Carson G, Quinn C, O'Connor T. The spiritual experience of high-risk pregnancy. Clinical research. 2006,36(1):63-70.

${ }^{19}$ Markovic M, Manderson L, Schaper H, Brennecke S. Maternal identity change as a consequence of antenatal hospitalization. Health Care Women Int. 2006;27(9):762776.

${ }^{20}$ Sittner B, De Frain J, Brage D. Effects of high-risk pregnancies on families. MCN. 2005;30(2):121-126.

${ }^{21}$ Silva E, Cordova F, Chachamovich JL, Záchia S. Percepções de um grupo de mulheres sobre a doença hipertensiva específica da gestação. Rev. Gaúcha Enferm. 2011;32(2):316-322.

${ }^{22}$ Leichtentritt R, Blumenthal N, Elyassi A, Rotmensch S. High-risk pregnancy and hospitalization: the women's voices. Health \& Social Work. 2005; 30 (1): 39-46.

${ }^{23}$ Richter M, Parkes Ch, Chaw-Kant J. Listening to the voices of hospitalized high-risk antepartum patients. JOGNN. 2007; 36(4);313-318.

${ }^{24}$ Barlow JH, Hainsworth J, Thornton S. Women's experiences of hospitalization with hypertension during pregnancy: Feeling a fraud. Journal of Reproductive and Infant Psychology. 2008;26(3):157-167.

${ }^{25}$ Oliveira V, Madeira A. Interagindo com a equipe multiprofissional: as interfaces da assistência na gestação de alto risco. Esc. Anna Nery. 2011;15(1):103-109. 